\title{
Tefritídeos endófagos (Diptera: Tephritidae) associados à Asteraceae em Chapecó, Santa Catarina
}

\author{
Sandra Mara Sabedot-Bordin ${ }^{1}$ \\ Gilson Marcos Bogus ${ }^{1}$ \\ Daiana Bampi ${ }^{1}$ \\ Flávio Roberto Mello Garcia ${ }^{2 *}$ \\ ${ }^{1}$ Universidade Comunitária da Região de Chapecó \\ PPG em Ciências Ambientais, Laboratório de Entomologia \\ ${ }^{2}$ Universidade Federal de Pelotas, Instituto de Biologia \\ Departamento de Zoologia e Genética, Laboratório de Ecologia de Insetos \\ Caixa Postal 35, CEP 96010-900, Pelotas - RS, Brasil \\ *Autor para correspondência \\ flavio.garcia@pq.cnpq.br
}

Submetido em 04/05/2010

Aceito para publicação em 04/10/2010

\section{Resumo}

O estudo foi realizado com o objetivo de inventariar a fauna de tefritídeos endófagos associados a capítulos de Asteraceae no município de Chapecó, Santa Catarina. As coletas de capítulos foram realizadas quinzenalmente no período de setembro de 2005 a setembro de 2006. Foram coletados capítulos de 37 espécies de Asteraceae, sendo registrados tefritídeos em 14 delas. Obteve-se dez espécies de tefritídeos endófagos. O gênero mais abundante foi Tomoplagia. Encontramos evidências de novos registros de associação entre tefritídeos endófagos e suas plantas hospedeiras.

Palavras-chave: Interação inseto-planta, Novos registros, Plantas hospedeiras, Tephritinae

\section{Abstract}

Endophagous tephritid flies (Diptera: Tephritidae) associated with Asteraceae in Chapecó, SC. This study was carried out aiming to make an inventory of the endophagous tephritid fauna associated with Asteraceae capitula in Chapecó town, Santa Catarina state. Samples of Asteraceae were taken from September 2005 to September 2006. Overall, we found 37 Asteraceae species; 14 of these presented tephritid species. Ten tephritid species were reared from the Asteraceae flowerheads. The most abundant genus was Tomoplagia. We found evidence of new associations between endophagous tephritids and host plants.

Key words: Insect-plant interactions, Host plants, New records, Tephritinae 


\section{Introdução}

Apesar dos tefritídeos serem comumente conhecidos como moscas-das-frutas, uma variedade de partes e tecidos vegetais é atacada, incluindo sementes, flores, caules, brotos, folhas e raízes. Os ovos são depositados em tecidos saudáveis da planta, onde as larvas alimentam-se, no entanto frutos e flores são as partes das plantas mais atacadas (GARCIA, 2009). A maioria dos representantes de Tephritinae alimentase exclusivamente de vegetais pertencentes à família Asteraceae (HEADRICK; GOEDEN, 1998). Para Santa Catarina, são conhecidas até o momento 81 espécies de Tephritidae incluídas em 24 gêneros (GARCIA, 2011).

Tephritidae é a família de Diptera com o maior número de registros de espécies associadas a Asteraceae no mundo (ZWÖLFER, 1987; FOOTE et al., 1993). As interações com as Asteraceae parecem ter sido uma das principais razões da grande diversificação dos tefritídeos (ZWÖLFER, 1987). No Brasil, ocorrem três famílias de dípteros (Tephritidae, Cecidomyiidae e Agromyzidae) e quatro de microlepidópteros (Tortricidae, Pterophoridae, Blastobasidae, Pyralidae, Gelechiidae) que atuam como endófagos de capítulos de Asteraceae, somando aproximadamente 120 espécies (LEWINSOHN, 1988; 1991).

Em se tratando de tefritídeos neotropicais as informações são escassas. O conhecimento mais avançado está para os grupos de importância econômica, especialmente sobre o gênero Anastrepha. Muitas espécies são importantes, atuando como agentes no controle biológico de plantas invasoras (NORRBOM, 2004). Existe pouca informação sobre biologia e taxonomia de grande número de espécies de tefritídeos associados à Asteraceae (FOOTE et al., 1993). No Brasil, os principais estudos realizados sobre a interação entre tefritídeos e Asteraceae são os realizados por: Lewinsohn (1991); Prado et al. (2002); Prado e Lewinsohn (2004); Almeida et al. (2006), Diniz et al. (2010), Fávero et al. (2010) havendo pouquíssimas informações até o momento sobre tal assunto em Santa Catarina.

Sendo assim, o presente trabalho tem como objetivo conhecer a fauna de tefritídeos endófagos associada à Asteraceae e relacionar as espécies hospedeiras ocorrentes no município de Chapecó, Santa Catarina.

\section{Material e Métodos}

As coletas de capítulos de Asteraceae foram realizadas quinzenalmente em cinco transectos aleatórios de $10 \mathrm{~m} \times 5 \mathrm{~m}$ em cada localidade por duas pessoas durante uma hora no período de setembro de 2005 a setembro de 2006 em Chapecó (2705'47'S; $\left.52^{\circ} 37^{\prime} 06^{\prime \prime} \mathrm{W}\right)$, Santa Catarina. As coletas foram realizadas nas seguintes bairros: Trevo, Linha Caravagio, Linha Rodeio Bonito, Presidente Médici, Centro, Santo Antonio, Linha Sede Trentin, Seminário, Marechal Borman, Campus da Unochapecó, Alta Floresta, Leopoldo Sander, Faxinal dos Rosas, Fazenda Zandavali e Linha São Roque.

Os capítulos foram acondicionados em potes de plástico transparente de 500 ou $1000 \mathrm{~mL}$ com tampa de tela, permanecendo sob temperatura de $25 \pm 3^{\circ} \mathrm{C}$, umidade relativa do ar de $70 \%$ e fotofase de $12 \mathrm{~h}$. Os potes eram inspecionados de um a quatro dias, para a retirada dos insetos adultos. Após quinze dias sem nenhuma emergência, a inspeção dos potes era encerrada.

Para cada espécie de Asteraceae foram feitas exsicatas para confirmação e testemunhos de identificação. Todas foram inicialmente separadas em morfoespécies e posteriormente identificadas. Todas as exsicatas foram enviadas para identificação por especialista e também confirmadas através da coleção de referência do Laboratório de Interação InsetosPlantas da UNICAMP. Os materiais testemunhos das plantas hospedeiras foram depositados no Herbário da UNOCHAPECÓ.

Os insetos foram fixados e montados em alfinete ou acondicionados em cápsulas de gelatina. A maioria dos insetos foi identificada por comparação com a coleção de referência do Laboratório de Interação Inseto-planta da Universidade Estadual de Campinas (UNICAMP). Todos os tefritídeos foram separados em morfoespécies, a maioria das quais identificada até espécie com o auxílio de chaves dicotômicas. Para gênero utilizaram-se chaves confeccionadas por Foote (1980). Para Neomyopites, 
utilizou-se chave de Steyskal (1979). Os espécimes de tefritídeos estão depositados na coleção do Laboratório de Entomologia da UNOCHAPECÓ.

\section{Resultados e Discussão}

Foram amostrados capítulos de 37 espécies de Asteraceae, distribuídas em nove tribos. Foram obtidas 10 espécies de tefritídeos incluídas em oito gêneros, sendo a maioria incluída no gênero Tomoplagia.

Foram obtidos espécimes de Dictyotrypeta em capítulos de Vernonanthura phosphorica (Vell.) H. Rob. (Vernonieae) (Tabela 1). Até o presente estudo, $V$. phosphorica não havia sido registrada como hospedeira de Dictyotrypeta. Prado et al. (2002) registraram sete morfoespécies de Dictyotrypeta, sendo que três usam Vernonieae como hospedeiras [incluindo D. atacta Hendel (1914)], duas usam Heliantheae e duas espécies usam hospedeiras das tribos Vernonieae e Mutisieae. Uma única espécie de Cecidochares, ainda não descrita, foi obtida em duas espécies da tribo Eupatorieae: $C$. laevigata e C. margaritensis (Tabela 1).

Registrou-se a ocorrência indivíduos de Dioxyna chilensis, todos em Bidens pilosa (Tabela 1). Esta planta é um novo registro de planta hospedeira para esta espécie. Dioxyna é um gênero de ampla distribuição,

TABELA 1: Tribos e espécies de Asteraceae hospedeiras e número de indivíduos de tefritídeos, distribuídos por espécies. Setembro de 2005 a setembro de 2006 em Chapecó, SC.

\begin{tabular}{|c|c|c|c|c|c|c|c|c|c|c|}
\hline Espécie & 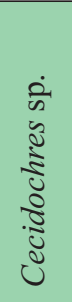 & 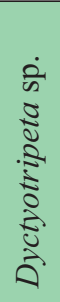 & 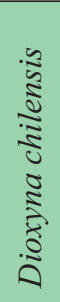 & 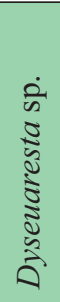 & 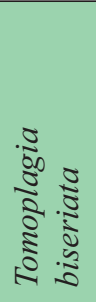 & 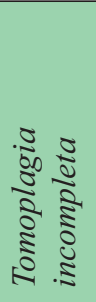 & 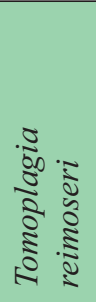 & 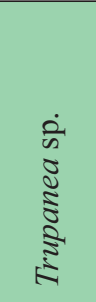 & 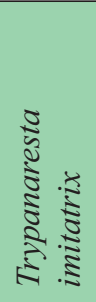 & 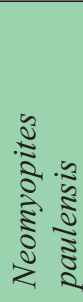 \\
\hline \multicolumn{11}{|l|}{$\begin{array}{l}\text { Eupatorieae } \\
\text { Ageratum conyzoides } \\
\text { Austroeupatorium inulae- } \\
\text { folium }\end{array}$} \\
\hline Chromolaena platensis & & & & & & & & 3.595 & & \\
\hline Chromolaena margaritensis & 12 & & & & & & & & & \\
\hline Chromolaena pedunculosa & & & & & & & & 18 & & \\
\hline \multicolumn{11}{|l|}{ Chromolaena squalida } \\
\hline Chromolaena laevigata & 872 & & & & & & & 77 & & 09 \\
\hline \multicolumn{11}{|l|}{ Mikania cordifolia } \\
\hline $\begin{array}{l}\text { Heliantheae } \\
\text { Bidens pilosa }\end{array}$ & & & 06 & & & & & & & \\
\hline $\begin{array}{l}\text { Vernonieae } \\
\text { Chrysolaena flexuosa }\end{array}$ & & & & & & 15 & & & & \\
\hline \multicolumn{11}{|l|}{ Lessingianthus glabratus } \\
\hline Vernonanthura discolor & & & & 38 & & & & & & \\
\hline Vernonanthura phosphorica & & 09 & & & 94 & & 392 & & & \\
\hline $\begin{array}{l}\text { Mutiseae } \\
\text { Gochnatia polymorpha }\end{array}$ & & & & & & & & & 13 & \\
\hline Total & 884 & 09 & 06 & 38 & 94 & 15 & 392 & 3.690 & 13 & 09 \\
\hline
\end{tabular}


com 11 espécies descritas (NORRBOM, 2004). No novo mundo, suas hospedeiras são principalmente espécies das tribos Heliantheae e Helenieae (PRADO et al., 2002). Neste mesmo estudo, D. chilensis Marcquart (1843) foi obtida de Isostigma sp. (Heliantheae) e Porophyllum ruderale (Jacq.) Cass. (Helenieae). As espécies Calea graminifolia (Heliantheae), P. riedelii (Helenieae) e Senecio oxyphyllus (Senecioneae) foram registradas hospedeiras ocasionais por Prado et al. (2002).

Obteve-se Dyseuaresta em Vernonanthura discolor (Vernonieae) (Tabela 1), coletada em borda de mata. $\mathrm{O}$ gênero não possui registros para Santa Catarina. Dyseuaresta é outro gênero neotropical pouco conhecido (FOOTE et al., 1993), até então, com 12 espécies descritas (NORRBOM, 2004). Estudo realizado por Prado et al. (2002) relata que D. mexicana Wiedemann (1830) foi obtida de Melanthera sp. Este gênero está associado primariamente com a tribo Heliantheae. Frías (1992) registrou D. impluviata Blanchard (1852) de Senecio, mas esta espécie pode ser confundida com Lamproxynella, um gênero cujos limites com Dyseuaresta não estão bem definidos.

Registraram-se indivíduos de $N$. paulensis em $C$. laevigata (Eupatorieae) (Tabela 1). De acordo com Foote et al. (1993), Neomyopites é considerado polifilético e apresenta ampla distribuição. Este foi proposto para incluir muitas das espécies do Novo Mundo, previamente colocadas em Urophora. Atualmente possui 28 espécies descritas (NORRBOM, 2004). Suas plantas hospedeiras conhecidas pertencem às tribos Astereae, Eupatorieae, Heliantheae e Liabeae (FREIDBERG e NORRBOM, 1999). Prado et al. (2002) registraram N. paulensis em 12 espécies, incluídas em cinco gêneros de Eupatorieae. Almeida et al. (2006) também registraram N. paulensis em C. laevigata e C. squalida.

O gênero neotropical Tomoplagia é um dos mais abundantes entre os tefritídeos que se desenvolvem em capítulos de Asteraceae no Brasil sendo composto por 59 espécies (NORRBOM, 2004) das quais pelo menos 25 ocorrem em nosso território (YOTOKO et al., 2005) e está primariamente associado à tribo Vernonieae. Das duas espécies que ocorrem nos Estados Unidos, T. obliqua (Say) está registrada para pelo menos sete espécies de Vernonieae, e T. cressoni Aczél para Trixis californica Kellog e Perezia microcephala (DC.) A. Gray (Mutisieae) (FOOTE et al., 1993). As principais plantas hospedeiras de todas as espécies de Tomoplagia pertencem a Vernonieae, com exceção de T. costalimai Aczél, a qual usa Trixis sp. (Mutiseae) e T. trivittata (Lutz e Lima), que utiliza espécies de Heliantheae (PRADO et al., 2002). Plantas das tribos Mutiseae e Heliantheae são ocasionalmente usadas por outras espécies de Tomoplagia.

Tomoplagia biseriata Loew (1873) e Tomoplagia reimoseri Hendel (1914), com 94 e 392 indivíduos, respectivamente, tiveram registro em $V$. phosphorica (Tabela 1). Essa planta foi coletada principalmente em área rural, ocorrendo em beira de estradas. $V$. phosphorica é um novo registro de hospedeira para $T$. biseriata. O primeiro registro de T. reimoseri para Santa Catarina foi feito por Sabedot et al. (2008).

As plantas hospedeiras de Tomoplagia pertencem à tribo Vernonieae, o mesmo fora constado também por Prado et al. (2002) (Tabela 1). Obteve-se Tomoplagia incompleta Williston (1896) em C. flexuosa (Tabela 1), sendo esta hospedeira coletada apenas em ambiente urbano, principalmente em terrenos baldios e beira de estradas. Esta pode ocasionalmente utilizar plantas das tribos Mutisieae e Eupatorieae, além de suas hospedeiras principais da tribo Vernonieae (PRADO e LEWINSOHN, 1994). Houve registro de T. incompleta em Vernonia velutina Glaz. por Lewinsohn (1988). Yotoko et al. (2005) relatam que a espécie pode ser considerada generalista por utilizar, além de vários hospedeiros da tribo Vernonieae, hospedeiros das tribos Mutisieae e eventualmente Eupatorieae.

Trypanaresta imitatrix Hering (1938) foi registrada apenas em G. polymorpha (Less.) Cabrera (Mutisieae), espécie arbórea, coletada em área urbana do município, em barrancos, sendo esta hospedeira principal. Porém, não há registros da ocorrência dessa espécie em $G$. polymorpha. Trypanaresta é um gênero de origem neotropical (NORRBOM et al., 1998), com 18 espécies descritas (NORRBOM, 2004). Obteve-se 3.690 indivíduos de Trupanea em Eupatorieae, associados às hospedeiras C. pedunculosa (Hook. \& Arn.) R.M. King \& H. Rob., C. platensis (Spreng.) H. Rob. e $C$. laevigata (Tabela 1). Prado et al. (2002) ainda registram 
este gênero em Mutisieae e as oito morfoespécies de Trupanea mostraram-se, na maioria, mais generalistas que as outras espécies de tefritídeos. A identificação de suas espécies é extremamente difícil, carecendo de uma revisão taxonômica adequada (FOOTE et al., 1993). O gênero Trupanea é um dos maiores entre os Tephritinae, sendo registradas 220 espécies (NORRBOM, 2004), 80 das quais na região neotropical (NORRBOM et al., 1998).

Na região neártica, os capítulos de Senecioneae são utilizados por pelo menos dez espécies de tefritídeos, dos gêneros Aciurina, Stenopa, Tephritis, Trupanea e Campiglossa (FOOTE et al., 1993). De acordo com Prado et al. (2002), tal padrão poderia ser explicado pela menor diversidade de espécies de Senecioneae no Brasil, sendo 83 espécies descritas, se comparada com as outras tribos importantes.

Em estudo realizado por Almeida et al. (2006), foi registrado Cecidochares sp. em C. laevigata o que é confirmado com o presente estudo. Cecidochares é um gênero exclusivamente neotropical, existem 12 espécies descritas (NORRBOM, 2004).

Dictyotrypeta também é um gênero exclusivamente neotropical com numerosas espécies ainda não descritas (FOOTE, 1980). Norrbom (2004) relata que atualmente estão descritas quatro espécies. Este gênero não possui registro para Santa Catarina. Quatro espécies de Dictyotrypeta foram registradas por Foote et al. (1993) em flores de Heliantheae. A biologia do gênero é pouco conhecida (PRADO et al., 2002).

As espécies de Asteraceae amostradas no município de Chapecó são hospedeiras de pelo menos 13 espécies de tefritídeos. A maioria das espécies de moscas foi obtida em capítulos de espécies da tribo Eupatorieae. Estudos visando a verificação de pupários destas espécies de tefritídeos em capítulos de Asteraceae são necessários para confirmar os novos registros de interações encontrados no presente estudo.

\section{Agradecimentos}

Desejamos externar nossos sinceros agradecimentos a UNOCHAPECÓ pelo auxílio com a bolsa de pesquisa a Sandra M. Sabedot-Bordin, ao CNPq pela Bolsa de Produtividade em Pesquisa de Flávio R. M. Garcia, ao Dr. Allen L. Norrbom do Systematic Entomology Laboratory (USDA) e ao Prof. Dr. Paulo Inácio Prado do Departamento de Ecologia do Instituto Biociências da Universidade de São Paulo, a toda a equipe do Laboratório de Interação Insetos-Plantas da UNICAMP pela confirmação das espécies de tefritídeos e ao Dr. Nelson Matzenbacher pela identificação de Asteraceae.

\section{Referências}

ALMEIDA, A. M.; FONSECA, C. R.; PRADO, P. I.; ALMEIDANETO, M.; DINIZ, S.; KUBOTA, U.;BRAUN, M. R.; RAIMUNDO, R. L. G.; ANJOS, L. A.; MENDONÇA, T. G.; FUTADA, S. M.; LEWINSOHN, T. M. Assemblages of endophagous insects on Asteraceae in São Paulo Cerrados. Neotropical Entomology, Piracicaba, v. 35, n. 4, p. 458-468, 2006.

DINIZ, S.; PRADO, P. I.; LEWINSOHN, T. M. Species richness in natural and disturbed habitats: Asteraceae and flower-head insects (Tephritidae: Diptera). Neotropical Entomology, Piracicaba, v. 39, n. 2, p. 163-171, 2010.

FÁVERO, K.; BORDIGNON, L.; VECCHI JR., K.; DINIZ, S. Efeito do tempo pós-queimada sobre comunidades de Tephritidae (Diptera) em áreas de cerrado na Chapada dos Guimarães, MT. Entomo Brasilis, Vassouras, v. 3, n. 2, p. 29-33, 2010.

FOOTE, R. H. Fruit fly Genera south of the United States. Washington: U. S. Government Technical Bulletin, 1980, 79 p.

FOOTE, R. H.; BLANC, F. L.; NORRBOM, A. L. Handbook of the fruit flies (Diptera: Tephritidae) of America and North of Mexico. Ithaca: Comstock Publishing Associates, 1993, $571 \mathrm{p}$.

FREIDBERG, A.; NORRBOM, A. L. A. generic reclassification and phylogeny of the tribe Myopitini (Tephritidae). In: ALUJA, M.; NORRBOM, A. L. (Ed.). Fruit flies (Tephritidae): phylogeny and evolution of behavior. Boca Raton: CRC Press, 1999, p. 581627.

FRÍAS, D.. Aspectos de la biologia evolutiva de especies de Tephritidae (Diptera). Acta Entomologica Chilena, v. 17, p. 6979, 1992.

GARCIA, F. R. M. Fruit fly: biological and ecological aspects. In: BANDEIRA, R. R. (Ed.). Current trends in fruit flies control on perennial crops and research prospects. Kerala: Transworld Research Network, 2009, p.1-35.

GARCIA, F. R. M. Distribuição geográfica no Brasil: Santa Catarina. In: MALAVASI, A.; ZUCCHI, R. A.; PEREIRA, R. C. (Ed.). Biologia, monitoramento, controle e distribuição de moscas-dasfrutas no Brasil. Ribeirão Preto: Editora Holos, 2011, no prelo.

HEADRICK, D. H.; GOEDEN, R. D. The biology of nonfrugivorous tephritid fruit flies. Annual Review of Entomology, Palo Alto, v. 43, p. 217-241, 1998.

LEWINSOHN, T. M. Composição e tamanho de faunas associadas a capítulos de compostas. 1988. 216 p. (Tese de Doutorado, Universidade Estadual de Campinas, Campinas) 
LEWINSOHN, T. M. Insects in flower heads of Asteraceae in Southeast Brazil: a tropical case study on species richness. In: PRICE, P. W.; LEWINSOHN, T. M.; FERNANDES, G. W.; BENSON, W. W. (Ed.). Plant-animal interactions: evolutionary ecology in tropical and temperate regions. Nova York: John Wiley \& Sons, 1991, p. 574-713.

NORRBOM, A. L. Fruit fly (Diptera: Tephritidae) species database. 2004. Disponível em: <http://www.sel.barc.usda. gov:591/diptera/Tephritidae/TephName/search.html>. Acesso em: 20 ago. 2008.

NORRBOM, A. L.; CARROLL, L. E.; FREIDBERG, A. Status of knowledge of Tephritid systematics. In: THOMPSON, F. C. (Ed.). Fruit fly expert identification system and systematic information. Leiden: Backhuys Publishers, 1998, p. 9-48.

PRADO, P. I. K. L.; LEWINSOHN, T. M. Genus Tomoplagia (Diptera, Tephritidae) in the Serra do Cipó, Minas Gerais, Brazil: host ranges and notes of taxonomic interest. Revista Brasileira de Entomologia, Curitiba, v. 38, n. 3, p. 669-680, 1994.

PRADO, P. I. K. L.; LEWINSOHN, T. M.; ALMEIDA, A. M.; NORRBOM, A. L.; BUYS, B. D.; MACEDO, A. C.; LOPES, M. B. The fauna of Tephritidae (Diptera) from capitula of Asteraceae in Brazil. Proceedings of the Entomological Society of Washington, Washington, v. 104, n. 4, p. 1007-1028, 2002.

SABEDOT, S. M.; GARCIA, F. R. M.; BOGUS, G. M.;
SAVARIS, M. Primeiro registro de Tomoplagia reimoseri Hendel e Xanthaciura mallochi Aczel (Diptera: Tephritidae) para o Estado de Santa Catarina. Ciência Rural, Santa Maria, v. 38, n. 6, p. 17621764. 2008.

STEYSKAL, G. C. Taxonomic studies on frut flies of the genus Urophora (Diptera: Tephritidae). Washington: The Entomological Society of Washington, 1979, 61p.

YOTOKO, K. S. C.; PRADO, P. I.; RUSSO, C. A. M.; SOLFERINI, $\mathrm{V}$. N. Testing the trend towards specialization in herbivore-host plant associations using a molecular phylogeny of Tomoplagia (Diptera: Tephritidae). Molecular Phylogenetics and Evolution, v. 35, n. 3, p. 701-711, 2005.

ZWÖLFER, H. Species richness, species packing and evolution in insect-plant systems. In: SCHULZE, E. D.; ZWÖLFER, H. (Ed.). Potentials and limitations of ecosystem analysis. Berlin: Springer-Verlag, 1987, p.301-319. 